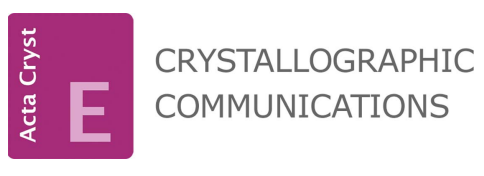

ISSN 2056-9890

Received 13 January 2016

Accepted 25 February 2016

Edited by A. J. Lough, University of Toronto, Canada

Keywords: crystal structure; sesquiterpene; isolation; Artemisia reticulata; antiproliferative property.

CCDC reference: 1455684

Supporting information: this article has supporting information at journals.iucr.org/e

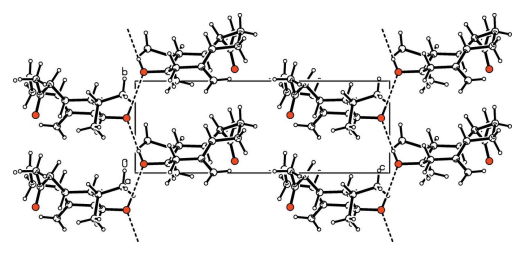

OPEN $\odot$ ACCESS

\section{Crystal structure of a bioactive sesquiterpene isolated from Artemisia reticulata}

\author{
A. K. Bauri, ${ }^{a}$ Sabine Foro ${ }^{b}$ and Nhu Quynh Nguyen Do ${ }^{\mathrm{c} *}$ \\ ${ }^{a}$ Bioorganic Division, Bhabha Atomic Research Centre, Trombay, Mumbai 85, India, ${ }^{\mathbf{b}}$ Clemens Schöpf-Institut für \\ Organische, Chemie und Biochemie, Technische Universität Darmstadt, Petersenstrasse 22, D-64287 Darmstadt, \\ Germany, and 'Accident \& Emergency Department, Franco, Vietnamese Hospital, 7-Nguyen, Luong Bang Street, \\ HoChiMinh City, Vietnam. *Correspondence e-mail: nguyendonhuquynh@yahoo.com
}

The title compound, $\mathrm{C}_{15} \mathrm{H}_{24} \mathrm{O}_{2}$ \{systematic name: 1-[6-hydroxy-7-(propan-2-yl)4-methylidene-2,3,3a,4,5,6,7,7a-octahydro- $1 H$-inden-1-yl]ethanone\} was isolated from $A$. reticulata by column chromatography over silica gel by gradient solvent elution. The molecule comprises a bicyclo[4.3.0]nonane ring bearing acetoxy, hydroxy and isopropyl substituents, and an exocyclic double bond on the cyclohexane ring. In the bicyclic skeleton, the cyclohexane ring adopts a chair conformation ring and the cyclopentane ring is in an envelope conformation. In the crystal, molecules are linked by $\mathrm{O}-\mathrm{H} \cdots \mathrm{O}$ hydrogen bonds, forming chains along [010]. These chains are cross-linked by $\mathrm{C}-\mathrm{H} \cdots \mathrm{O}$ hydrogen bonds.

\section{Chemical context}

The title compound is a natural product, which has been isolated from the Indian herb $A$. reticulata by column chromatography over silica gel. A. reticulata (family: Asteraceae) is a traditional herb which has many applications in folklore medicine for conventional therapy against several diseases such as malaria (Klayman et al., 1984; Malagon et al., 1997; Newton \& White, 1999), cancer (Efferth et al., 2001; Lai et al., 1995), cardiovascular (Guantai et al., 1999), vasodilatory (Walker, 1996), hepatitis (Aniya et al., 2000) and diabetes (Iriadam et al., 2006). It is found as a constituent in many ayurvedic or herbal drug preparations such as forkolin and Afsanteen in Indian traditional medicinal systems (Nadkarni, 1954; Satyavati et al., 1987; Subramoniam et al., 1996; Drury, 1978). The Artemisia species are a rich source of bioactive sesquiterpenenoids (Klayman et al., 1984) such as artemisinin, artemisin etc. Artimisinin and artemisin are secondary metabolites isolated from herbs of the species $A$. аппиа (Klayman, 1985) belonging to the sesquiterpene class. The title molecule possesses antiplasmodial activity and it is now under clinical trial for the treatment of malaria. Our group are currently searching for artemisin, artemisinin or their analogues from other varieties of Artemisia species and as part of these studies, the structure of the title compound is now reported.

\section{Structural commentary}

The molecular structure of the title compound is shown in Fig. 1. The compound comprises fused cyclohexane and cyclopentane rings. It has been substantiated by a positive LB test (Liebermann Burchard Test), which indicates that it 
belongs to the sesquiterpene class. The compound is soluble in chloroform but has poor solubility in methanol.

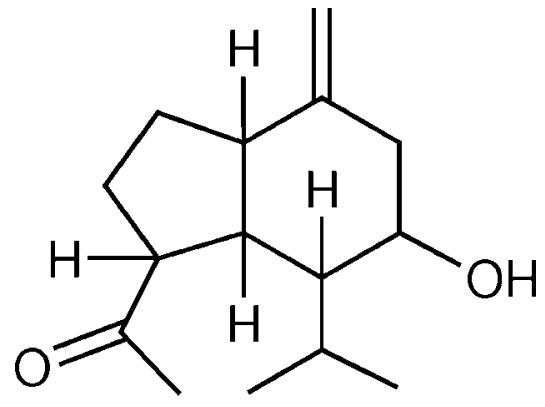

The bicyclic skeleton contains one acetyl group at atom $\mathrm{C} 1$ of the cyclopentane ring, one isopropyl group and one hydroxyl group located at atoms C6 and C7 in the cyclohexane ring. An exocyclic olefinic double bond is located between atoms $\mathrm{C} 9$ and $\mathrm{C} 15$ and attached to the cyclohexane ring. The torsion angles $\mathrm{C} 3-\mathrm{C} 4-\mathrm{C} 5-\mathrm{C} 6$ and $\mathrm{C} 9-\mathrm{C} 4-\mathrm{C} 5-\mathrm{C} 1$ of $-169.2(3)$ and $-170.9(3)^{\circ}$, respectively, describe the geometry at the junction of the two rings. The $\mathrm{C} 7-\mathrm{C} 6-\mathrm{C} 5$ and C9-C4-C5 angles are 107.3 (2) and $109.2(3)^{\circ}$, respectively.

\section{Supramolecular features}

In the crystal, molecules are linked by $\mathrm{O}-\mathrm{H} \cdots \mathrm{O}$ hydrogen bonds, forming chains along [010] (Table 1 and Fig. 2). These chains are cross-linked by weak $\mathrm{C}-\mathrm{H}$. . O hydrogen bonds.

\section{Database survey}

A search of Cambridge Structural Database (CSD, Version 5.36, last update May 2015; Groom \& Allen, 2015) found only one molecule, Pulioplopane A (15-hydroxy-10 (14)-oplopen-

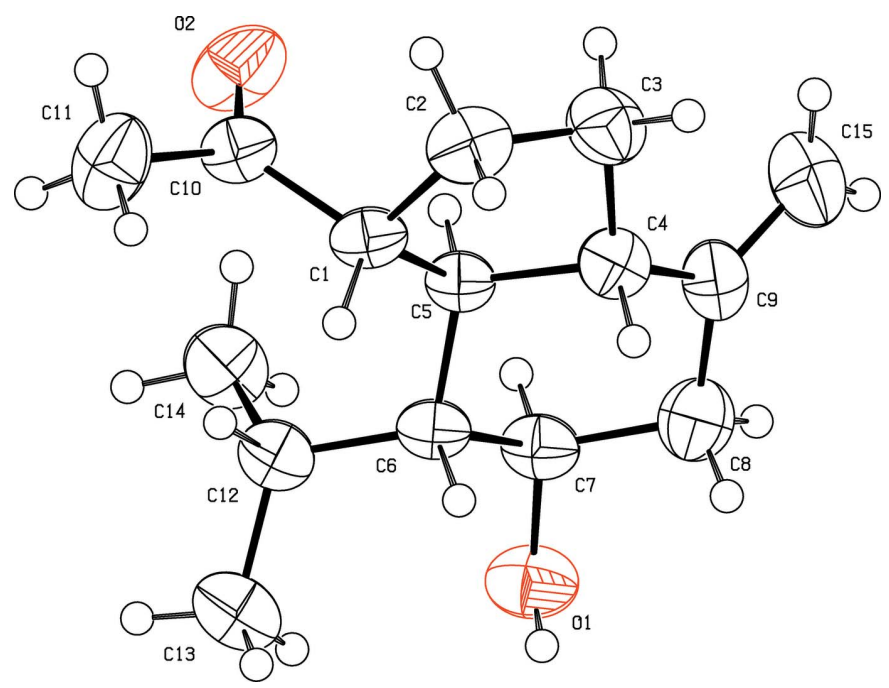

Figure 1

The molecular structure of the title compound, showing $50 \%$ probability displacement ellipsoids for non- $\mathrm{H}$ atoms.
Table 1

Hydrogen-bond geometry $\left(\AA{ }^{\circ}\right)$.

\begin{tabular}{lllll}
\hline$D-\mathrm{H} \cdots A$ & $D-\mathrm{H}$ & $\mathrm{H} \cdots A$ & $D \cdots A$ & $D-\mathrm{H} \cdots A$ \\
\hline $\mathrm{O}^{1}-\mathrm{H} 1 O \cdots \mathrm{O} 1^{\mathrm{i}}$ & 0.82 & 2.11 & $2.927(4)$ & 175 \\
$\mathrm{C}^{1}-\mathrm{H} 11 C \cdots \mathrm{O} 2^{\mathrm{ii}}$ & 0.96 & 2.53 & $3.430(6)$ & 157 \\
\hline
\end{tabular}

Symmetry codes: (i) $-x+1, y+\frac{1}{2},-z$; (ii) $x, y+1, z$.

4-one; Triana et al., 2005) that has a similar structural skeleton to the title sesquitertene although it is is unrelated in a biochemical sense.

\section{Synthesis and crystallization}

The title sesquiterpene was isolated as colourless solid from the methanol extract of $A$. reticulata by chromatography over silica gel with a mixture of ethyl acetate and hexane with a gradient elution followed by preparative thin layer chromatography. Crystals were obtained after recrystallization three times from ethyl acetate:hexane (1:4) at room temperature by the slow evaporation method. Bioassay of this molecule has been conducted against human ovarian cancer cell line A 2780 and revealed that it possessed significant antiproliferative activity (unpublished results).

\section{Refinement}

Crystal data, data collection and structure refinement details are summarized in Table 2. $\mathrm{H}$ atoms were placed in calculated positions with $\mathrm{C}-\mathrm{H}=0.93-0.98 \AA$ and $\mathrm{O}-\mathrm{H}=0.82 \AA$ and refined in a riding-motion approximation with $U_{\text {iso }}(\mathrm{U})=$ $1.2 U_{\text {eq }}(\mathrm{C}, \mathrm{O})$. No Friedel pairs were collected therefore the absolute configuration could not be determined from the $\mathrm{X}$-ray data and the assignment is arbitrary.

\section{Acknowledgements}

The authors thank Professor Dr Hartmut, FG Strukturforschung, Material-und Geowissenschaften, Technische Universität Darmstadt, Petersenstress 23, 64287 Darmstadt, and Professor Kingston, Department of Chemistry, M/C 0212, Virginia Polytechnic Institute and State University, Blacks-

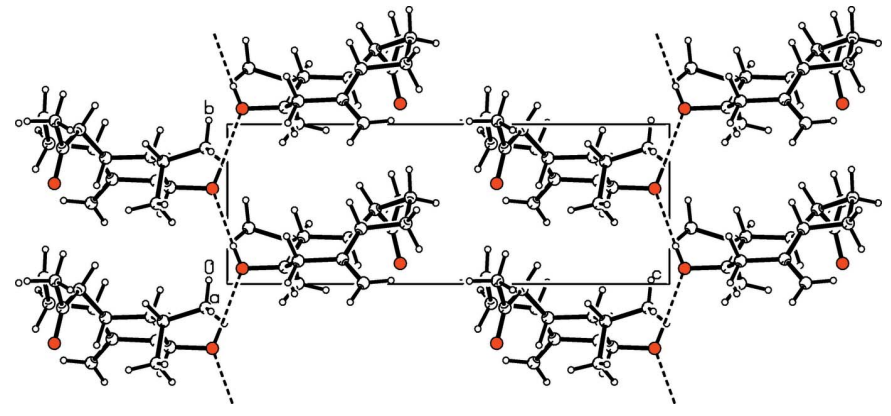

Figure 2

Part of the crystal structure of the title compound, with hydrogen bonds shown as dashed lines. 
burg, Virginia 24061, USA, for their kind co-operation to measure diffraction data for the title compound and to carry out an antiproliferative bioassay against cancer cell lines.

\section{References}

Aniya, Y., Shimabukuro, M., Shimoji, M., Kohatsu, M., Gyamfi, M. A., Miyagi, C., Kunii, D., Takayama, F. \& Egashira, T. (2000). Biol. Pharm. Bull. 23, 309-312.

Drury, C. H. (1978). The useful Plants of India: with notices of their chief value in commerce, medicine and the arts. London: William $\mathrm{H}$. Allen \& Co.

Efferth, T., Dunstan, H., Sauerbrey, A., Miyachi, H. \& Chitambar, C. R. (2001). Int. J. Oncol. 18, 767-773.

Groom, C. R. \& Allen, F. H. (2014). Angew. Chem. Int. Ed. 53, 662671.

Guantai, A. N. \& Addae-Mensah, I. (1999). Pharm. Biol. 37, 351-356.

Iriadam, M., Musa, D., Gumushan, H. \& Baba, F. (2006). J. Cell. Mol. Biol. 5, 19-24.

Klayman, D. L. (1985). Science, 228, 1049-1055.

Klayman, D. L., Lin, A., Acton, N., Scovill, J. P., Hoch, J. M., Milhous, W. K., Theoharides, A. D. \& Dobek, A. S. (1984). J. Nat. Prod. 47, 715-717.

Lai, H. \& Singh, N. P. (1995). Cancer Lett. 91, 41-46.

Malagon, F., Vazquez, J., Delgado, G. \& Ruiz, A. (1997). Parassitolgia, 39, 3-7.

Nadkarni, A. K. (1954). Indian Materia Medica, 3rd ed. Bombay: Popular Prakashan.

Newton, P. \& White, N. (1999). Annu. Rev. Med. 50, 179-192.

Nonius (1993). CAD-4-PC Software. Enraf-Nonius, Delft, The Netherlands.

Satyavati, G. V., Gupta, A. \& Tandon, N. (1987). In Medicinal Plants of India. New Delhi: ICMR.

Sheldrick, G. M. (2008). Acta Cryst. A64, 112-122.

Spek, A. L. (2009). Acta Cryst. D65, 148-155.

Stoe \& Cie (1987). REDU4. Stoe \& Cie GmbH, Darmstadt, Germany.
Table 2

Experimental details.

\begin{tabular}{ll}
\hline Crystal data & \\
Chemical formula & $\mathrm{C}_{15} \mathrm{H}_{24} \mathrm{O}_{2}$ \\
$M_{\mathrm{r}}$ & 236.34 \\
Crystal system, space group & Monoclinic, $P 2_{1}$ \\
Temperature $(\mathrm{K})$ & 299 \\
$a, b, c(\AA)$ & $8.849(4), 5.336(1), 14.994(5)$ \\
$\beta\left({ }^{\circ}\right.$ & $99.21(2)$ \\
$V\left(\AA^{3}\right)$ & $698.9(4)$ \\
$Z$ & 2 \\
Radiation type & $\mathrm{Cu} \mathrm{K \alpha}$ \\
$\mu\left(\mathrm{mm}^{-1}\right)$ & 0.56 \\
Crystal size $(\mathrm{mm})$ & $0.50 \times 0.18 \times 0.15$ \\
& \\
Data collection & \\
Diffractometer & Enraf-Nonius CAD-4 \\
No. of measured, independent and & $1916,1392,1260$ \\
$\quad$ observed $[I>2 \sigma(I)]$ reflections & \\
$R_{\text {int }}$ & 0.052 \\
$(\text { sin } \theta / \lambda)_{\text {max }}\left(\AA^{-1}\right)$ & 0.597 \\
Refinement & \\
$R\left[F^{2}>2 \sigma\left(F^{2}\right)\right], w R\left(F^{2}\right), S$ & \\
No. of reflections & $0.060,0.164,1.10$ \\
No. of parameters & 1392 \\
No. of restraints & 154 \\
$\mathrm{H}$-atom treatment & 1 \\
$\Delta \rho_{\text {max }}, \Delta \rho_{\text {min }}\left(\mathrm{e} \AA^{-3}\right)$ & $\mathrm{H}-$ atom parameters constrained \\
\hline
\end{tabular}

Computer programs: $C A D-4-P C$ (Enraf-Nonius, 1993), REDU4 (Stoe \& Cie, 1987), SHELXS97 and SHELXL97 (Sheldrick, 2008) and PLATON (Spek, 2009).

Subramoniam, A., Pushpangadan, P., Rajasekharan, S., Evans, D. A., Latha, P. G. \& Valsaraj, R. (1996). J. Ethnopharmacol. 50, 13-17. Triana, J., López, M., Pérez, F. J., González-Platas, J., Quintana, J., Estévez, F., León, F. \& Bermejo, J. (2005). J. Nat. Prod. 68, 523-531. Walker, A. F. (1996). Biologist, 43, 177-180. 


\section{supporting information}

Acta Cryst. (2016). E72, 460-462 [https://doi.org/10.1107/S2056989016003236]

\section{Crystal structure of a bioactive sesquiterpene isolated from Artemisia reticulata}

\section{A. K. Bauri, Sabine Foro and Nhu Quynh Nguyen Do}

Computing details

Data collection: $C A D-4-P C$ (Enraf-Nonius, 1993); cell refinement: $C A D-4-P C$ (Enraf-Nonius, 1993); data reduction: REDU4 (Stoe \& Cie, 1987); program(s) used to solve structure: SHELXS97 (Sheldrick, 2008); program(s) used to refine structure: SHELXL97 (Sheldrick, 2008); molecular graphics: PLATON (Spek, 2009); software used to prepare material for publication: SHELXL97 (Sheldrick, 2008).

1-[6-Hydroxy-7-(propan-2-yl)-4-methylidene-2,3,3a,4,5,6,7,7a-octahydro-1H-inden-1-yl] ethanone

Crystal data

$\mathrm{C}_{15} \mathrm{H}_{24} \mathrm{O}_{2}$

$M_{r}=236.34$

Monoclinic, $P 2_{1}$

Hall symbol: $\mathrm{P} 2 \mathrm{yb}$

$a=8.849$ (4) $\AA$

$b=5.336(1) \AA$

$c=14.994(5) \AA$

$\beta=99.21(2)^{\circ}$

$V=698.9(4) \AA^{3}$

$Z=2$

\section{Data collection}

Enraf-Nonius CAD-4 diffractometer

Radiation source: fine-focus sealed tube Graphite monochromator $\omega / 2 \theta$ scans

1916 measured reflections 1392 independent reflections 1260 reflections with $I>2 \sigma(I)$

\section{Refinement}

Refinement on $F^{2}$

Least-squares matrix: full

$R\left[F^{2}>2 \sigma\left(F^{2}\right)\right]=0.060$

$w R\left(F^{2}\right)=0.164$

$S=1.10$

1392 reflections

154 parameters

1 restraint

Primary atom site location: structure-invariant direct methods
$F(000)=260$

$D_{\mathrm{x}}=1.123 \mathrm{Mg} \mathrm{m}^{-3}$

$\mathrm{Cu} K \alpha$ radiation, $\lambda=1.54180 \AA$

Cell parameters from 25 reflections

$\theta=5.5-27.1^{\circ}$

$\mu=0.56 \mathrm{~mm}^{-1}$

$T=299 \mathrm{~K}$

Rod, colourless

$0.50 \times 0.18 \times 0.15 \mathrm{~mm}$

$R_{\text {int }}=0.052$

$\theta_{\text {max }}=67.0^{\circ}, \theta_{\text {min }}=3.0^{\circ}$

$h=-10 \rightarrow 3$

$k=0 \rightarrow 6$

$l=-17 \rightarrow 17$

3 standard reflections every $120 \mathrm{~min}$

intensity decay: $1.0 \%$

Secondary atom site location: difference Fourier map

Hydrogen site location: inferred from neighbouring sites

$\mathrm{H}$-atom parameters constrained

$w=1 /\left[\sigma^{2}\left(F_{\mathrm{o}}^{2}\right)+(0.1147 P)^{2}+0.0812 P\right]$

where $P=\left(F_{\mathrm{o}}{ }^{2}+2 F_{\mathrm{c}}{ }^{2}\right) / 3$

$(\Delta / \sigma)_{\max }<0.001$

$\Delta \rho_{\max }=0.46 \mathrm{e} \AA^{-3}$

$\Delta \rho_{\min }=-0.22$ e $\AA^{-3}$ 


\section{Special details}

Geometry. All esds (except the esd in the dihedral angle between two 1.s. planes) are estimated using the full covariance matrix. The cell esds are taken into account individually in the estimation of esds in distances, angles and torsion angles; correlations between esds in cell parameters are only used when they are defined by crystal symmetry. An approximate (isotropic) treatment of cell esds is used for estimating esds involving l.s. planes.

Refinement. Refinement of $\mathrm{F}^{2}$ against ALL reflections. The weighted R-factor $\mathrm{wR}$ and goodness of fit $\mathrm{S}$ are based on $\mathrm{F}^{2}$, conventional R-factors $\mathrm{R}$ are based on $\mathrm{F}$, with $\mathrm{F}$ set to zero for negative $\mathrm{F}^{2}$. The threshold expression of $\mathrm{F}^{2}$ \&gt;

$2 \operatorname{sigma}\left(\mathrm{F}^{2}\right)$ is used only for calculating R-factors(gt) etc. and is not relevant to the choice of reflections for refinement. Rfactors based on $\mathrm{F}^{2}$ are statistically about twice as large as those based on F, and R- factors based on ALL data will be even larger.

Fractional atomic coordinates and isotropic or equivalent isotropic displacement parameters $\left(\AA^{2}\right)$

\begin{tabular}{|c|c|c|c|c|}
\hline & $x$ & $y$ & $z$ & $U_{\text {iso }} * / U_{\text {eq }}$ \\
\hline $\mathrm{O} 1$ & $0.5467(3)$ & $0.0974(5)$ & 0.03391 (14) & $0.0580(7)$ \\
\hline $\mathrm{H} 1 \mathrm{O}$ & 0.5259 & 0.2380 & 0.0135 & $0.070^{*}$ \\
\hline $\mathrm{O} 2$ & $0.9664(3)$ & $0.1293(5)$ & $0.3910(2)$ & $0.0680(8)$ \\
\hline $\mathrm{C} 1$ & $0.8020(3)$ & $0.4666(6)$ & $0.33401(19)$ & $0.0401(7)$ \\
\hline $\mathrm{H} 1$ & 0.8184 & 0.6168 & 0.2992 & $0.048^{*}$ \\
\hline $\mathrm{C} 2$ & $0.7177(4)$ & $0.5402(8)$ & $0.4141(2)$ & 0.0539 (9) \\
\hline $\mathrm{H} 2 \mathrm{~A}$ & 0.7821 & 0.5069 & 0.4715 & $0.065^{*}$ \\
\hline $\mathrm{H} 2 \mathrm{~B}$ & 0.6912 & 0.7167 & 0.4112 & $0.065^{*}$ \\
\hline $\mathrm{C} 3$ & $0.5742(4)$ & $0.3792(9)$ & $0.4035(2)$ & $0.0568(9)$ \\
\hline $\mathrm{H} 3 \mathrm{~A}$ & 0.5943 & 0.2188 & 0.4335 & $0.068^{*}$ \\
\hline H3B & 0.4923 & 0.4633 & 0.4277 & $0.068 *$ \\
\hline $\mathrm{C} 4$ & $0.5350(3)$ & $0.3473(7)$ & $0.3022(2)$ & $0.0446(7)$ \\
\hline $\mathrm{H} 4$ & 0.5020 & 0.5109 & 0.2765 & $0.053^{*}$ \\
\hline $\mathrm{C} 5$ & 0.6918 (3) & $0.2887(5)$ & $0.27514(17)$ & $0.0355(6)$ \\
\hline H5 & 0.7191 & 0.1174 & 0.2952 & $0.043 *$ \\
\hline C6 & $0.6880(3)$ & $0.2967(5)$ & $0.17229(17)$ & $0.0370(7)$ \\
\hline H6 & 0.6501 & 0.4629 & 0.1518 & $0.044 *$ \\
\hline $\mathrm{C} 7$ & $0.5672(4)$ & $0.1059(6)$ & $0.13003(18)$ & $0.0439(7)$ \\
\hline $\mathrm{H} 7$ & 0.6039 & -0.0600 & 0.1517 & $0.053^{*}$ \\
\hline $\mathrm{C} 8$ & $0.4103(4)$ & $0.1446(9)$ & $0.1602(2)$ & $0.0590(10)$ \\
\hline $\mathrm{H} 8 \mathrm{~A}$ & 0.3654 & 0.2991 & 0.1341 & $0.071 *$ \\
\hline $\mathrm{H} 8 \mathrm{~B}$ & 0.3429 & 0.0081 & 0.1371 & $0.071^{*}$ \\
\hline C9 & $0.4206(4)$ & $0.1563(8)$ & $0.2604(2)$ & $0.0508(8)$ \\
\hline $\mathrm{C} 10$ & 0.9549 (4) & $0.3515(7)$ & $0.3722(2)$ & $0.0431(7)$ \\
\hline C11 & $1.0900(4)$ & $0.5203(9)$ & $0.3893(3)$ & $0.0671(11)$ \\
\hline H11A & 1.1664 & 0.4637 & 0.3552 & $0.081 *$ \\
\hline H11B & 1.1313 & 0.5185 & 0.4526 & $0.081^{*}$ \\
\hline $\mathrm{H} 11 \mathrm{C}$ & 1.0594 & 0.6878 & 0.3713 & $0.081^{*}$ \\
\hline $\mathrm{C} 12$ & 0.8473 (4) & $0.2670(6)$ & 0.1447 (2) & $0.0447(8)$ \\
\hline H12 & 0.9160 & 0.3774 & 0.1847 & $0.054^{*}$ \\
\hline $\mathrm{C} 13$ & $0.8571(5)$ & $0.3509(9)$ & $0.0487(2)$ & $0.0604(10)$ \\
\hline H13A & 0.8294 & 0.5246 & 0.0418 & $0.072 *$ \\
\hline H13B & 0.7882 & 0.2524 & 0.0067 & $0.072 *$ \\
\hline $\mathrm{H} 13 \mathrm{C}$ & 0.9598 & 0.3286 & 0.0371 & $0.072 *$ \\
\hline
\end{tabular}




$\begin{array}{lllll}\text { C14 } & 0.9125(5) & 0.0040(8) & 0.1601(3) & 0.0613(10) \\ \text { H14A } & 0.8454 & -0.1135 & 0.1251 & 0.074^{*} \\ \text { H14B } & 0.9217 & -0.0377 & 0.2230 & 0.074^{*} \\ \text { H14C } & 1.0116 & -0.0027 & 0.1419 & 0.074^{*} \\ \text { C15 } & 0.3418(5) & 0.0067(11) & 0.3076(3) & 0.0708(12) \\ \text { H15A } & 0.2759 & -0.1134 & 0.2780 & 0.085^{*} \\ \text { H15B } & 0.3528 & 0.0225 & 0.3701 & 0.085^{*}\end{array}$

Atomic displacement parameters $\left(\AA^{2}\right)$

\begin{tabular}{lllllll}
\hline & $U^{11}$ & $U^{22}$ & $U^{33}$ & $U^{12}$ & $U^{13}$ & $U^{23}$ \\
\hline O1 & $0.0792(15)$ & $0.0505(15)$ & $0.0419(11)$ & $-0.0110(14)$ & $0.0024(10)$ & $-0.0077(11)$ \\
O2 & $0.0730(17)$ & $0.0337(14)$ & $0.0893(19)$ & $0.0019(14)$ & $-0.0110(13)$ & $0.0124(14)$ \\
C1 & $0.0543(16)$ & $0.0251(14)$ & $0.0392(13)$ & $-0.0004(13)$ & $0.0022(12)$ & $0.0020(12)$ \\
C2 & $0.067(2)$ & $0.045(2)$ & $0.0479(16)$ & $0.0059(17)$ & $0.0046(14)$ & $-0.0101(16)$ \\
C3 & $0.0614(19)$ & $0.063(2)$ & $0.0489(17)$ & $0.0040(18)$ & $0.0176(14)$ & $-0.0047(18)$ \\
C4 & $0.0501(16)$ & $0.0376(17)$ & $0.0472(16)$ & $0.0042(15)$ & $0.0112(12)$ & $-0.0022(14)$ \\
C5 & $0.0451(14)$ & $0.0226(14)$ & $0.0386(14)$ & $-0.0002(12)$ & $0.0059(11)$ & $0.0015(12)$ \\
C6 & $0.0513(16)$ & $0.0226(14)$ & $0.0369(13)$ & $0.0002(13)$ & $0.0064(11)$ & $0.0013(12)$ \\
C7 & $0.0570(17)$ & $0.0330(17)$ & $0.0410(14)$ & $-0.0066(15)$ & $0.0052(13)$ & $-0.0001(14)$ \\
C8 & $0.0527(18)$ & $0.062(3)$ & $0.0603(19)$ & $-0.010(2)$ & $0.0022(14)$ & $-0.004(2)$ \\
C9 & $0.0423(15)$ & $0.049(2)$ & $0.0626(18)$ & $-0.0012(16)$ & $0.0135(13)$ & $-0.0002(17)$ \\
C10 & $0.0516(17)$ & $0.0324(16)$ & $0.0438(15)$ & $-0.0007(14)$ & $0.0026(12)$ & $-0.0008(14)$ \\
C11 & $0.0541(19)$ & $0.047(2)$ & $0.095(3)$ & $-0.0055(18)$ & $-0.0023(18)$ & $-0.002(2)$ \\
C12 & $0.0551(17)$ & $0.0307(16)$ & $0.0492(17)$ & $-0.0035(15)$ & $0.0108(13)$ & $-0.0007(14)$ \\
C13 & $0.078(2)$ & $0.052(2)$ & $0.0559(19)$ & $-0.004(2)$ & $0.0248(17)$ & $-0.0006(18)$ \\
C14 & $0.070(2)$ & $0.042(2)$ & $0.077(2)$ & $0.0144(19)$ & $0.0264(18)$ & $0.0068(19)$ \\
C15 & $0.060(2)$ & $0.076(3)$ & $0.080(2)$ & $-0.014(2)$ & $0.0229(18)$ & $-0.004(2)$ \\
& & & & & &
\end{tabular}

Geometric parameters $\left(\AA,{ }^{\circ}\right)$

\begin{tabular}{llll}
\hline $\mathrm{O} 1-\mathrm{C} 7$ & $1.424(3)$ & $\mathrm{C} 7-\mathrm{C} 8$ & $1.542(5)$ \\
$\mathrm{O} 1-\mathrm{H} 1 \mathrm{O}$ & 0.8200 & $\mathrm{C} 7-\mathrm{H} 7$ & 0.9800 \\
$\mathrm{O} 2-\mathrm{C} 10$ & $1.219(5)$ & $\mathrm{C} 8-\mathrm{C} 9$ & $1.493(5)$ \\
$\mathrm{C} 1-\mathrm{C} 10$ & $1.513(4)$ & $\mathrm{C} 8-\mathrm{H} 8 \mathrm{~A}$ & 0.9700 \\
$\mathrm{C} 1-\mathrm{C} 5$ & $1.535(4)$ & $\mathrm{C} 8-\mathrm{H} 8 \mathrm{~B}$ & 0.9700 \\
$\mathrm{C} 1-\mathrm{C} 2$ & $1.562(5)$ & $\mathrm{C} 9-\mathrm{C} 15$ & $1.335(6)$ \\
$\mathrm{C} 1-\mathrm{H} 1$ & 0.9800 & $\mathrm{C} 10-\mathrm{C} 11$ & $1.486(5)$ \\
$\mathrm{C} 2-\mathrm{C} 3$ & $1.521(6)$ & $\mathrm{C} 11-\mathrm{H} 11 \mathrm{~A}$ & 0.9600 \\
$\mathrm{C} 2-\mathrm{H} 2 \mathrm{~A}$ & 0.9700 & $\mathrm{C} 11-\mathrm{H} 11 \mathrm{~B}$ & 0.9600 \\
$\mathrm{C} 2-\mathrm{H} 2 \mathrm{~B}$ & 0.9700 & $\mathrm{C} 11-\mathrm{H} 11 \mathrm{C}$ & 0.9600 \\
$\mathrm{C} 3-\mathrm{C} 4$ & $1.513(4)$ & $\mathrm{C} 12-\mathrm{C} 14$ & $1.521(5)$ \\
$\mathrm{C} 3-\mathrm{H} 3 \mathrm{~A}$ & 0.9700 & $\mathrm{C} 12-\mathrm{C} 13$ & $1.523(5)$ \\
$\mathrm{C} 3-\mathrm{H} 3 \mathrm{~B}$ & 0.9700 & $\mathrm{C} 12-\mathrm{H} 12$ & 0.9800 \\
$\mathrm{C} 4-\mathrm{C} 9$ & $1.501(5)$ & $\mathrm{C} 13-\mathrm{H} 13 \mathrm{~A}$ & 0.9600 \\
$\mathrm{C} 4-\mathrm{C} 5$ & $1.539(4)$ & $\mathrm{C} 13-\mathrm{H} 13 \mathrm{~B}$ & 0.9600 \\
$\mathrm{C} 4-\mathrm{H} 4$ & 0.9800 & $\mathrm{C} 13-\mathrm{H} 13 \mathrm{C}$ & 0.9600 \\
$\mathrm{C} 5-\mathrm{C} 6$ & $1.538(3)$ & $\mathrm{C} 14-\mathrm{H} 14 \mathrm{~A}$ & 0.9600
\end{tabular}




\begin{tabular}{|c|c|c|c|}
\hline $\mathrm{C} 5-\mathrm{H} 5$ & 0.9800 & $\mathrm{C} 14-\mathrm{H} 14 \mathrm{~B}$ & 0.9600 \\
\hline $\mathrm{C} 6-\mathrm{C} 7$ & $1.537(4)$ & $\mathrm{C} 14-\mathrm{H} 14 \mathrm{C}$ & 0.9600 \\
\hline $\mathrm{C} 6-\mathrm{C} 12$ & $1.540(4)$ & $\mathrm{C} 15-\mathrm{H} 15 \mathrm{~A}$ & 0.9300 \\
\hline $\mathrm{C} 6-\mathrm{H} 6$ & 0.9800 & C15-H15B & 0.9300 \\
\hline $\mathrm{C} 7-\mathrm{O} 1-\mathrm{H} 1 \mathrm{O}$ & 109.5 & $\mathrm{C} 6-\mathrm{C} 7-\mathrm{H} 7$ & 106.8 \\
\hline $\mathrm{C} 10-\mathrm{C} 1-\mathrm{C} 5$ & $114.5(3)$ & $\mathrm{C} 8-\mathrm{C} 7-\mathrm{H} 7$ & 106.8 \\
\hline $\mathrm{C} 10-\mathrm{C} 1-\mathrm{C} 2$ & $108.6(2)$ & $\mathrm{C} 9-\mathrm{C} 8-\mathrm{C} 7$ & $112.9(3)$ \\
\hline $\mathrm{C} 5-\mathrm{C} 1-\mathrm{C} 2$ & $105.1(2)$ & $\mathrm{C} 9-\mathrm{C} 8-\mathrm{H} 8 \mathrm{~A}$ & 109.0 \\
\hline $\mathrm{C} 10-\mathrm{C} 1-\mathrm{H} 1$ & 109.5 & $\mathrm{C} 7-\mathrm{C} 8-\mathrm{H} 8 \mathrm{~A}$ & 109.0 \\
\hline $\mathrm{C} 5-\mathrm{C} 1-\mathrm{H} 1$ & 109.5 & $\mathrm{C} 9-\mathrm{C} 8-\mathrm{H} 8 \mathrm{~B}$ & 109.0 \\
\hline $\mathrm{C} 2-\mathrm{C} 1-\mathrm{H} 1$ & 109.5 & $\mathrm{C} 7-\mathrm{C} 8-\mathrm{H} 8 \mathrm{~B}$ & 109.0 \\
\hline $\mathrm{C} 3-\mathrm{C} 2-\mathrm{C} 1$ & $105.8(3)$ & $\mathrm{H} 8 \mathrm{~A}-\mathrm{C} 8-\mathrm{H} 8 \mathrm{~B}$ & 107.8 \\
\hline $\mathrm{C} 3-\mathrm{C} 2-\mathrm{H} 2 \mathrm{~A}$ & 110.6 & $\mathrm{C} 15-\mathrm{C} 9-\mathrm{C} 8$ & $123.8(4)$ \\
\hline $\mathrm{C} 1-\mathrm{C} 2-\mathrm{H} 2 \mathrm{~A}$ & 110.6 & $\mathrm{C} 15-\mathrm{C} 9-\mathrm{C} 4$ & $124.0(4)$ \\
\hline $\mathrm{C} 3-\mathrm{C} 2-\mathrm{H} 2 \mathrm{~B}$ & 110.6 & $\mathrm{C} 8-\mathrm{C} 9-\mathrm{C} 4$ & $112.2(3)$ \\
\hline $\mathrm{C} 1-\mathrm{C} 2-\mathrm{H} 2 \mathrm{~B}$ & 110.6 & $\mathrm{O} 2-\mathrm{C} 10-\mathrm{C} 11$ & $121.0(3)$ \\
\hline $\mathrm{H} 2 \mathrm{~A}-\mathrm{C} 2-\mathrm{H} 2 \mathrm{~B}$ & 108.7 & $\mathrm{O} 2-\mathrm{C} 10-\mathrm{C} 1$ & $121.3(3)$ \\
\hline $\mathrm{C} 4-\mathrm{C} 3-\mathrm{C} 2$ & $102.8(3)$ & $\mathrm{C} 11-\mathrm{C} 10-\mathrm{C} 1$ & $117.7(3)$ \\
\hline $\mathrm{C} 4-\mathrm{C} 3-\mathrm{H} 3 \mathrm{~A}$ & 111.2 & $\mathrm{C} 10-\mathrm{C} 11-\mathrm{H} 11 \mathrm{~A}$ & 109.5 \\
\hline $\mathrm{C} 2-\mathrm{C} 3-\mathrm{H} 3 \mathrm{~A}$ & 111.2 & $\mathrm{C} 10-\mathrm{C} 11-\mathrm{H} 11 \mathrm{~B}$ & 109.5 \\
\hline $\mathrm{C} 4-\mathrm{C} 3-\mathrm{H} 3 \mathrm{~B}$ & 111.2 & $\mathrm{H} 11 \mathrm{~A}-\mathrm{C} 11-\mathrm{H} 11 \mathrm{~B}$ & 109.5 \\
\hline $\mathrm{C} 2-\mathrm{C} 3-\mathrm{H} 3 \mathrm{~B}$ & 111.2 & $\mathrm{C} 10-\mathrm{C} 11-\mathrm{H} 11 \mathrm{C}$ & 109.5 \\
\hline $\mathrm{H} 3 \mathrm{~A}-\mathrm{C} 3-\mathrm{H} 3 \mathrm{~B}$ & 109.1 & $\mathrm{H} 11 \mathrm{~A}-\mathrm{C} 11-\mathrm{H} 11 \mathrm{C}$ & 109.5 \\
\hline $\mathrm{C} 9-\mathrm{C} 4-\mathrm{C} 3$ & $121.8(3)$ & $\mathrm{H} 11 \mathrm{~B}-\mathrm{C} 11-\mathrm{H} 11 \mathrm{C}$ & 109.5 \\
\hline $\mathrm{C} 9-\mathrm{C} 4-\mathrm{C} 5$ & $109.2(3)$ & $\mathrm{C} 14-\mathrm{C} 12-\mathrm{C} 13$ & $109.7(3)$ \\
\hline $\mathrm{C} 3-\mathrm{C} 4-\mathrm{C} 5$ & $102.5(2)$ & $\mathrm{C} 14-\mathrm{C} 12-\mathrm{C} 6$ & $113.3(3)$ \\
\hline $\mathrm{C} 9-\mathrm{C} 4-\mathrm{H} 4$ & 107.5 & $\mathrm{C} 13-\mathrm{C} 12-\mathrm{C} 6$ & $114.6(3)$ \\
\hline $\mathrm{C} 3-\mathrm{C} 4-\mathrm{H} 4$ & 107.5 & $\mathrm{C} 14-\mathrm{C} 12-\mathrm{H} 12$ & 106.2 \\
\hline $\mathrm{C} 5-\mathrm{C} 4-\mathrm{H} 4$ & 107.5 & $\mathrm{C} 13-\mathrm{C} 12-\mathrm{H} 12$ & 106.2 \\
\hline $\mathrm{C} 1-\mathrm{C} 5-\mathrm{C} 6$ & $118.0(2)$ & $\mathrm{C} 6-\mathrm{C} 12-\mathrm{H} 12$ & 106.2 \\
\hline $\mathrm{C} 1-\mathrm{C} 5-\mathrm{C} 4$ & $103.8(2)$ & $\mathrm{C} 12-\mathrm{C} 13-\mathrm{H} 13 \mathrm{~A}$ & 109.5 \\
\hline $\mathrm{C} 6-\mathrm{C} 5-\mathrm{C} 4$ & $112.5(2)$ & $\mathrm{C} 12-\mathrm{C} 13-\mathrm{H} 13 \mathrm{~B}$ & 109.5 \\
\hline $\mathrm{C} 1-\mathrm{C} 5-\mathrm{H} 5$ & 107.3 & $\mathrm{H} 13 \mathrm{~A}-\mathrm{C} 13-\mathrm{H} 13 \mathrm{~B}$ & 109.5 \\
\hline $\mathrm{C} 6-\mathrm{C} 5-\mathrm{H} 5$ & 107.3 & $\mathrm{C} 12-\mathrm{C} 13-\mathrm{H} 13 \mathrm{C}$ & 109.5 \\
\hline $\mathrm{C} 4-\mathrm{C} 5-\mathrm{H} 5$ & 107.3 & $\mathrm{H} 13 \mathrm{~A}-\mathrm{C} 13-\mathrm{H} 13 \mathrm{C}$ & 109.5 \\
\hline $\mathrm{C} 7-\mathrm{C} 6-\mathrm{C} 5$ & $107.3(2)$ & $\mathrm{H} 13 \mathrm{~B}-\mathrm{C} 13-\mathrm{H} 13 \mathrm{C}$ & 109.5 \\
\hline $\mathrm{C} 7-\mathrm{C} 6-\mathrm{C} 12$ & $115.3(3)$ & $\mathrm{C} 12-\mathrm{C} 14-\mathrm{H} 14 \mathrm{~A}$ & 109.5 \\
\hline $\mathrm{C} 5-\mathrm{C} 6-\mathrm{C} 12$ & $113.1(2)$ & $\mathrm{C} 12-\mathrm{C} 14-\mathrm{H} 14 \mathrm{~B}$ & 109.5 \\
\hline $\mathrm{C} 7-\mathrm{C} 6-\mathrm{H} 6$ & 106.9 & $\mathrm{H} 14 \mathrm{~A}-\mathrm{C} 14-\mathrm{H} 14 \mathrm{~B}$ & 109.5 \\
\hline $\mathrm{C} 5-\mathrm{C} 6-\mathrm{H} 6$ & 106.9 & $\mathrm{C} 12-\mathrm{C} 14-\mathrm{H} 14 \mathrm{C}$ & 109.5 \\
\hline $\mathrm{C} 12-\mathrm{C} 6-\mathrm{H} 6$ & 106.9 & $\mathrm{H} 14 \mathrm{~A}-\mathrm{C} 14-\mathrm{H} 14 \mathrm{C}$ & 109.5 \\
\hline $\mathrm{O} 1-\mathrm{C} 7-\mathrm{C} 6$ & $113.9(2)$ & $\mathrm{H} 14 \mathrm{~B}-\mathrm{C} 14-\mathrm{H} 14 \mathrm{C}$ & 109.5 \\
\hline $\mathrm{O} 1-\mathrm{C} 7-\mathrm{C} 8$ & $109.0(2)$ & $\mathrm{C} 9-\mathrm{C} 15-\mathrm{H} 15 \mathrm{~A}$ & 120.0 \\
\hline $\mathrm{C} 6-\mathrm{C} 7-\mathrm{C} 8$ & $112.9(3)$ & C9-C15-H15B & 120.0 \\
\hline $\mathrm{O} 1-\mathrm{C} 7-\mathrm{H} 7$ & 106.8 & $\mathrm{H} 15 \mathrm{~A}-\mathrm{C} 15-\mathrm{H} 15 \mathrm{~B}$ & 120.0 \\
\hline $\mathrm{C} 10-\mathrm{C} 1-\mathrm{C} 2-\mathrm{C} 3$ & $-116.4(3)$ & $\mathrm{C} 5-\mathrm{C} 6-\mathrm{C} 7-\mathrm{C} 8$ & $52.9(3)$ \\
\hline
\end{tabular}




$\begin{array}{llll}\mathrm{C} 5-\mathrm{C} 1-\mathrm{C} 2-\mathrm{C} 3 & 6.5(4) & \mathrm{C} 12-\mathrm{C} 6-\mathrm{C} 7-\mathrm{C} 8 & 179.9(3) \\ \mathrm{C} 1-\mathrm{C} 2-\mathrm{C} 3-\mathrm{C} 4 & -31.5(4) & \mathrm{O} 1-\mathrm{C} 7-\mathrm{C} 8-\mathrm{C} 9 & -179.9(3) \\ \mathrm{C} 2-\mathrm{C} 3-\mathrm{C} 4-\mathrm{C} 9 & 166.7(3) & \mathrm{C} 6-\mathrm{C} 7-\mathrm{C} 8-\mathrm{C} 9 & -124.2(4) \\ \mathrm{C} 2-\mathrm{C} 3-\mathrm{C} 4-\mathrm{C} 5 & \mathrm{C} 7-\mathrm{C} 8-\mathrm{C} 9-\mathrm{C} 15 & 53.1(4) \\ \mathrm{C} 10-\mathrm{C} 1-\mathrm{C} 5-\mathrm{C} 6 & \mathrm{C} 7-\mathrm{C} 8-\mathrm{C} 9-\mathrm{C} 4 & 2.1(6) \\ \mathrm{C} 2-\mathrm{C} 1-\mathrm{C} 5-\mathrm{C} 6 & \mathrm{C} 3-\mathrm{C} 4-\mathrm{C} 9-\mathrm{C} 15 & 121.1(4) \\ \mathrm{C} 10-\mathrm{C} 1-\mathrm{C} 5-\mathrm{C} 4 & 145.9(3) & \mathrm{C} 5-\mathrm{C} 4-\mathrm{C} 9-\mathrm{C} 15 & -175.3(3) \\ \mathrm{C} 2-\mathrm{C} 1-\mathrm{C} 5-\mathrm{C} 4 & \mathrm{C} 3-\mathrm{C} 4-\mathrm{C} 9-\mathrm{C} 8 & -56.3(4) \\ \mathrm{C} 9-\mathrm{C} 4-\mathrm{C} 5-\mathrm{C} 1 & \mathrm{C} 5-\mathrm{C} 4-\mathrm{C} 9-\mathrm{C} 8 & -31.7(5) \\ \mathrm{C} 3-\mathrm{C} 4-\mathrm{C} 5-\mathrm{C} 1 & \mathrm{C} 5-\mathrm{C} 1-\mathrm{C} 10-\mathrm{O} 2 & 85.3(4) \\ \mathrm{C} 9-\mathrm{C} 4-\mathrm{C} 5-\mathrm{C} 6 & \mathrm{C} 2-\mathrm{C} 1-\mathrm{C} 10-\mathrm{O} 2 & -92.5(4) \\ \mathrm{C} 3-\mathrm{C} 4-\mathrm{C} 5-\mathrm{C} 6 & \mathrm{C} 5-\mathrm{C} 1-\mathrm{C} 10-\mathrm{C} 11 & -53.3(4) \\ \mathrm{C} 1-\mathrm{C} 5-\mathrm{C} 6-\mathrm{C} 7 & \mathrm{C} 2-\mathrm{C} 1-\mathrm{C} 10-\mathrm{C} 11 & 70.7(4) \\ \mathrm{C} 4-\mathrm{C} 5-\mathrm{C} 6-\mathrm{C} 7 & 60.4(3) & \mathrm{C} 7-\mathrm{C} 6-\mathrm{C} 12-\mathrm{C} 14 & 73.6(4) \\ \mathrm{C} 1-\mathrm{C} 5-\mathrm{C} 6-\mathrm{C} 12 & -169.2(3) & \mathrm{C} 5-\mathrm{C} 6-\mathrm{C} 12-\mathrm{C} 14 & -162.4(3) \\ \mathrm{C} 4-\mathrm{C} 5-\mathrm{C} 6-\mathrm{C} 12 & -179.0(3) & \mathrm{C} 7-\mathrm{C} 6-\mathrm{C} 12-\mathrm{C} 13 & \\ \mathrm{C} 5-\mathrm{C} 6-\mathrm{C} 7-\mathrm{O} 1 & -58.0(3) & \mathrm{C} 5-\mathrm{C} 6-\mathrm{C} 12-\mathrm{C} 13 & \\ \mathrm{C} 12-\mathrm{C} 6-\mathrm{C} 7-\mathrm{O} 1 & 52.8(3) & & \end{array}$

Hydrogen-bond geometry $\left(\AA,{ }^{\circ}\right)$

\begin{tabular}{lllll}
\hline$D-\mathrm{H} \cdots A$ & $D-\mathrm{H}$ & $\mathrm{H} \cdots A$ & $D \cdots A$ & $D-\mathrm{H} \cdots A$ \\
\hline $\mathrm{O} 1-\mathrm{H} 1 O \cdots \mathrm{O} 1^{\mathrm{i}}$ & 0.82 & 2.11 & $2.927(4)$ & 175 \\
$\mathrm{C} 11-\mathrm{H} 11 C \cdots \mathrm{O} 2^{\mathrm{ii}}$ & 0.96 & 2.53 & $3.430(6)$ & 157
\end{tabular}

Symmetry codes: (i) $-x+1, y+1 / 2,-z$; (ii) $x, y+1, z$. 